\title{
Observed variety of timescales in variability of P Cygni
}

I. Kolka, Tartu Astrophysical Observatory, EE2444 Tõravere, Estonia

1. P Cygni is a LBV (B1 Ia + ) which exhibits photometric and spectroscopic variability.

2. We present observational data from two periods (1981-82 and 1989-90) pointing to three different variability timescales.

3. Data sources:

a) spectroscopy - our observations at Tartu and results published earlier (Markova, Kolka 1989),

b) photometry - our compilation (Kolka, de Groot, Percy 1993).

4. The features measured:

a) the brightness $(\mathrm{m}(\mathrm{V}))$ in $\mathrm{V}$-filter,

b) the residual intensity $\left(R_{c}\right)$ and the radial velocity $\left(V_{r}\right.$ in $\left.\mathrm{km} / \mathrm{s}\right)$ of the absorption trough in chosen spectral lines.

5. Important quasi-stabil variability timescales (see Fig.1, Fig.2) in our data:

a) cycle-length $230 \ldots 240$ days in the variations of $R_{c}$ in Balmer-line $\mathrm{H} 9$ correlated with the $V_{r}$-oscillations in the same line. The $\mathrm{m}(\mathrm{V})$-curve has regularly local maxima near the moment of residual intensity minimum. The deepening of the absorption trough is related to the higher average level of the brightness.

b) cycle-length around 50 days in the behaviour of $R_{c}$ and $V_{r}$ in the line HeI3820 (most pronounced in the period 1981 - 82 (Fig.1) when minima in $R_{c}$ occurred as a rule together with maxima in $V_{r}$ ). This timescale-value is often observable in the lightcurve, too (see also Percy et al. 1988).

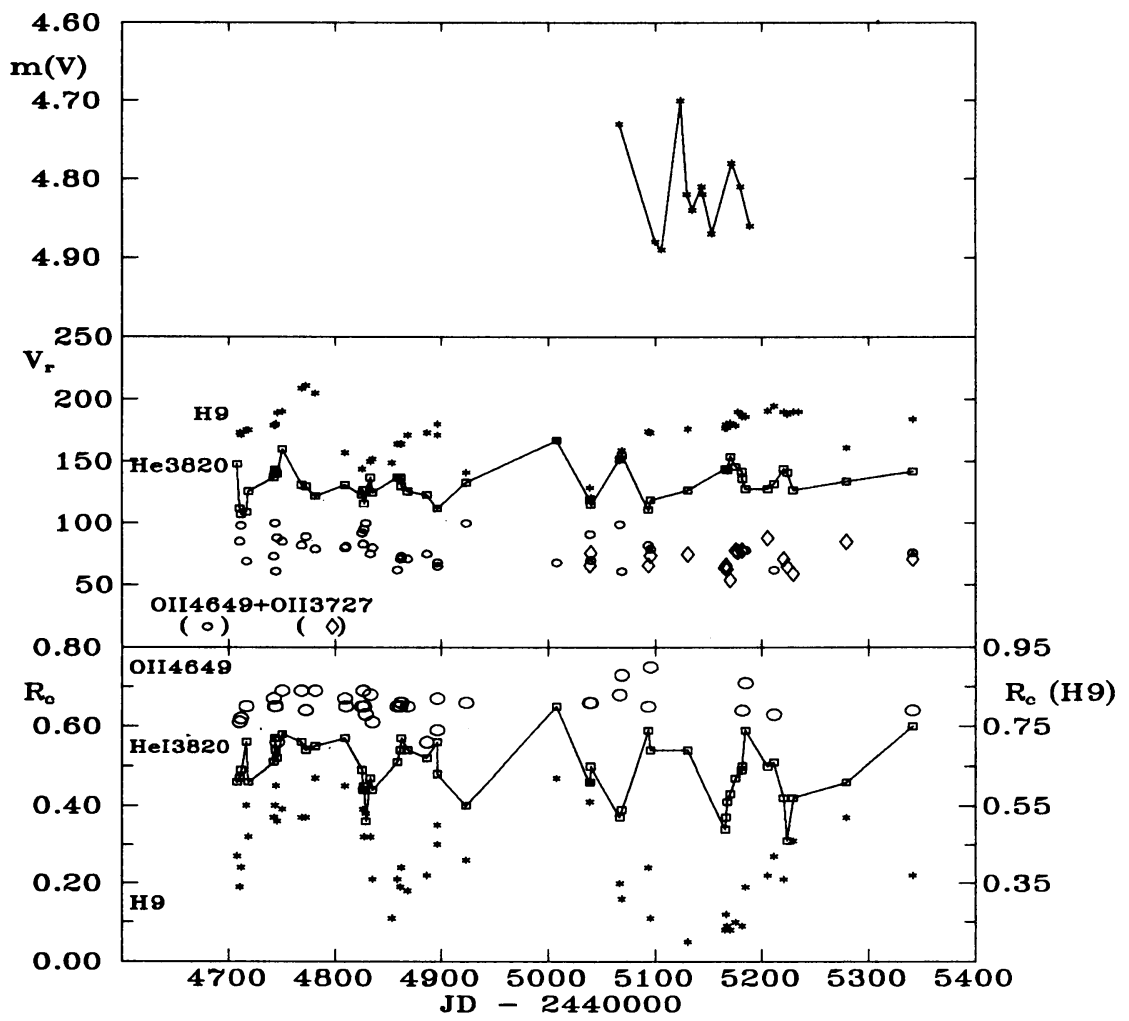

Fig.1 The variability curves in $1981-82$ 


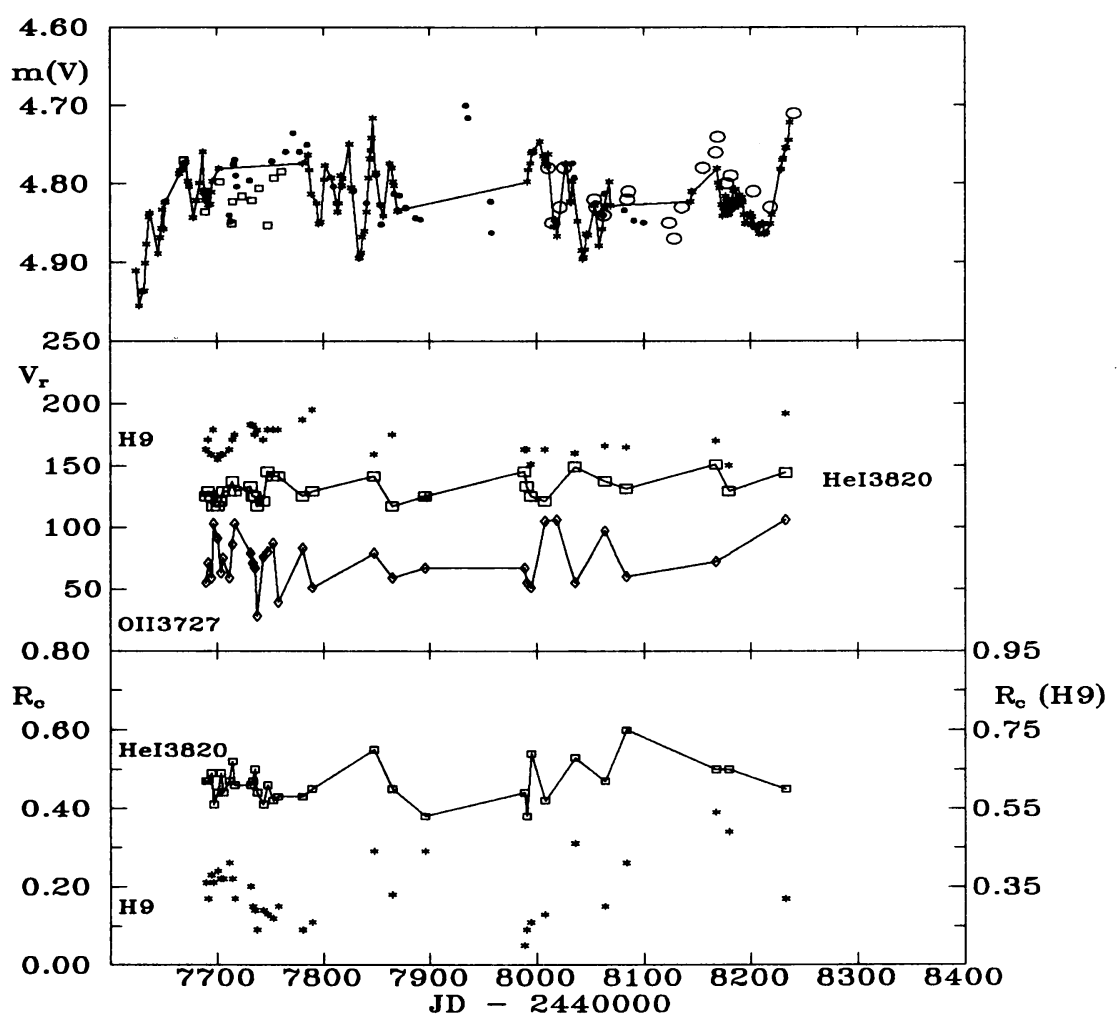

Fig. 2 The variability curves in $1989-90$

c) cycle-length $15 . .20$ days is common in $\mathrm{V}_{\mathrm{r}}$-variability of weak lines (e.g. OII3727) and in lightcurve data with sufficient time resolution.

6. We believe the time-dependent intensity of matter outflow is responsible for all variability cycles.

The longest cycle (over 200 days) reflects the swinging of the envelope between the states of lower and higher density which may be caused by the bistability of the stellar wind (Pauldrach, Puls 1990).

The shortest timescale (15...20 days) can be in connection with pulsations - having probably the nonradial character (Percy, Welch 1983) - which excite the inhomogeneous non-isotropic mass ejection in the form of " blobs" as it was suggested on the basis of spectropolarimetric measurements (Taylor et al. 1991). The alternative way to get the changing density in the essential volume of the envelope is in this case the changing birthrate and/or the varying dimensions of "blobs" due to the interaction of slightly different periods generated by the pulsational instability.

The cycle with the medium length can be fitted to this scenario by incorporating the rotational modulation of the asymmetrical outflow of "blobs" (the estimated rotation period of P Cygni is $\sim 50$ days (Percy et al. 1988)).

\section{References}

Kolka I., de Groot M., Percy J.R., 1993, (in preparation)

Markova N., Kolka I., 1989, An Atlas of Spectral Line Profiles of P Cygni in 1981-83, Tartu Teated nr.103.

Pauldrach A.W.A., Puls J., 1990, Astron. Astrophys. 237,409-424.

Percy J.R., Welch D.L., 1983, PASP 95,491-505.

Percy J.R. et al., 1988, Astron. Astrophys. 191,248-252.

Taylor M. et al., 1991, Astron. J. 102,1197-1206. 